\title{
Topiramate in migraine progression
}

\author{
Luigi Ruiz $\cdot$ Delfina Ferrandi
}

Received: 20 April 2009/Accepted: 26 September 2009/Published online: 17 October 2009

(C) Springer-Verlag 2009

\begin{abstract}
Increasing evidence shows that migraine, typically considered as an episodic disease, is a chronic and, in some patients, progressive disorder. Among neuromodulators used for migraine prevention, topiramate has a high level of evidence-based efficacy. Through its wide range of mechanisms of action topiramate increases the activation threshold resulting in neuronal stabilization and thereby reducing cortical neurons hyperexcitability, which is believed to be an important electrophysiological feature underlying the pathogenesis of epilepsy and migraine. Recent studies show that migraineurs have subclinical structural brain changes and persistent alteration of pain perception, in some cases correlated with the duration of the disease and the frequency of attacks that might play a role in the transformation of episodic migraine to chronic forms. An early and prolonged preventive treatment might reduce the risk of such transformation. Recent evidence suggests that topiramate, by reducing migraine frequency and use of acute medication, may prevent the negative progression of migraine. Furthermore, two recently completed multicenter, randomised, placebo-controlled trials have shown that treatment with topiramate $100 \mathrm{mg}$ /day is effective and well tolerated in patients already progressed to chronic migraine and difficult to treat conditions associated with medication-overuse. Topiramate seems to be a preventive treatment, which might be able to act at different levels of the migraine cycle: reduction of frequency in episodic migraine, prevention, and treatment of chronic migraine.
\end{abstract}

\footnotetext{
L. Ruiz $(\bowtie) \cdot$ D. Ferrandi

SOC Neurologia-Azienda Ospedaliera SS.

Antonio e Biagio, via Venezia 16, Alessandria, Italy

e-mail:1ruiz@ospedale.al.it

D. Ferrandi

e-mail: dferrandi@ospedale.al.it
}

Keywords Topiramate - Chronic migraine . Preventive treatment $\cdot$ Migraine progression

\section{Introduction}

In recent years, our knowledge about the pathophysiology of migraine has improved leading to the concept that the disease, typically expressed by episodic manifestations, is, actually, a chronic, and in a subgroup of patients, progressive disorder. Many authors indicated that the disease could lead to anatomic and functional changes in the central nervous system [1].

In patients with migraine, a preventive therapy should reduce frequency and severity of attacks in order to reduce acute medication use, to improve quality of life and to reduce the risk of progression to a chronic form. Bigal and Lipton [2] explained mechanisms of migraine progression from different points of view: clinical, physiological, and anatomic. They also proposed a model to address bidirectional migraine transitions from one form to another (i.e., low frequency episodic migraine, high frequency episodic migraine, chronic migraine-CM) with transition rates modelled as a function of demographic, environmental and genetic risk factors. Among risk factors, migraine frequency and acute medication overuse are believed as important targets contributing to the transformation from episodic to chronic migraine.

Topiramate is a neuromodulator approved for the use in epilepsy and migraine prevention in USA and in many European and world markets. In the present review, we summarize the most reliable hypothesis concerning pathophysiology of migraine, topiramate mechanisms of action, moreover we discuss its role in preventing and treating chronic migraine. 


\section{Pathophysiology}

Cortical spreading depression (CSD), discovered by Leão in 1944 [3], is implicated in the pathogenesis of migraine headaches [4, 5]. CSD is characterized by a slowly spreading wave of neuronal and glial depolarization lasting approximately $1 \mathrm{~min}$ that develops within brain areas such as cerebral cortex, cerebellum, or hippocampus after electrical or chemical stimulation. The pivotal event in the generation and propagation of CSD is a marked decrease of neuronal membrane resistance associated with a massive increase in extracellular $\mathrm{K}^{+}$and an increase in intracellular $\mathrm{Na}^{+}$and $\mathrm{Ca}^{2+}$. Various stimuli trigger CSD including direct cortical trauma, exposure to high concentrations of excitatory amino acids or $\mathrm{K}^{+}$, direct electrical stimulation, inhibition of $\mathrm{Na}^{+} / \mathrm{K}^{+}$-ATPase, and energy failure.

A link between CSD and migraine pathogenesis has been hypothesized for more than 40 years [4-7]. Many recent studies using magnetoencephalography or highfield strength, high-resolution magnetic resonance imaging (MRI) established a link between migraine aura and CSD $[8,9]$.

An interesting possibility is that CSD or CSD-like events may also play a role in the pathophysiology of migraine without aura (MWoA), perhaps after CSD is generated within brain regions that are clinically silent [10].

A number of recent studies in migraineurs $[11,12]$ show subclinical structural brain changes, going from progressive cellular damage in nociceptive systems to diffuse white matter (WM) loss, multifocal WM lesions, and ischemic stroke. They found these changes only in a small subset of patients. A study with Diffusion Tension Imaging (DTI), more sensitive than conventional MRI, detects subtle WM changes that are not necessarily ischemic [13]. In this study, Granziera and colleagues demonstrated that cortical and subcortical structures involved in motion perception differ from migraineurs and people without migraine. Chronic dysfunction of these areas may explain interictal findings of motion processing deficits in migraineurs, whereas episodic dysfunction might contribute to the initiation of an attack. Data indicate that structural changes are present in cortical regions in migraine patients, even outside attacks, that migraine with aura (MWA) and MWoA shares the same abnormalities, and that the changes are present in areas where CSD most probably develops. It seems likely that MWA and MWoA are two phenotypes sharing a common pathophysiological substrate and that asymptomatic CSD may occur in some patients.

The finding of morphometric changes in the brains of migraineurs may be of fundamental importance because migraine has so far been considered a condition with abnormal brain function but completely normal brain structure.

Chronic systemic treatment with migraine prophylactic drugs suppresses CSD as a potential explanation of their efficacy in migraine prophylaxis. Using two complementary paradigms, Ayata [10] showed that chronic treatment with topiramate, valproate, propranolol, amitriptyline, or methysergide rendered rat cerebral cortex more resistant to CSD and longer treatments produced stronger CSD inhibition.

The risk of developing chronic headache appears, at least in part, linked to heredity and genetics. The relative risk for developing chronic daily headache $(\mathrm{CDH})$ in first-degree relatives was found to be 2.1-3.9 times greater than the known risk within the general population [14]. Hypotheses for the pathogenesis of $\mathrm{CM}$ have included conditions of low central neuronal serotonin (5-HT), disregulation of neuronal 5-HT receptors, hyperexcitability of central pain pathways, low central $\beta$-endorphin levels, and dysfunction of NMDA receptors [15]. There are several lines of evidence to suggest that repeated episodes of migraine headache are associated with changes in CNS structure or function. Through specialized (MRI) brain imaging techniques, Welch and colleagues [1] demonstrated abnormal non-heme iron deposition in the periaqueductal gray matter of the rostral brainstem in migraineurs but not in control subjects. The amount of iron deposited was positively correlated with the subjects' lifetime duration of migraine. It was hypothesized that these structural changes occur through iron-catalyzed free radical cell damage "accentuated by repeated episodes of hyperoxia" during migraine attacks. These findings suggest that repeated attacks of migraine result in iron accumulation in the antinociceptive systems of the brainstem, potentially causing or acting as a marker for impaired pain modulation.

Clinical observations and a growing body of evidence indicate that chronic headache is associated with persistent hyperalgesia and allodynia [16]. It has been hypothesized that prolonged nociceptive input might not only incite sensitization of central pain pathways but also cause neural plasticity in these pathways by promoting the transformation of episodic headache to chronic forms of headache [15]. This hypothesis appears to be supported by recent epidemiological and clinic-based studies that sought to identify risk factors for this phenotypic transformation of headache presentation. Headache frequency was found to be an important and substantial risk factor for headache transformation [17]. A baseline headache frequency of four to five headache days per month was identified as a threshold for initiating an exponential increase in the risk of transformation from episodic headache to chronic forms, including CM [18]. These findings can imply that reduction in migraine attack severity, through aggressive use of acute migraine treatments, and decrease of migraine frequency with preventive migraine treatments may reduce the risk of neural plasticity and consequential transformation to a more chronic, refractory, and disabling migraine pattern. Acute medication overuse is also considered an important risk factor leading to the development of $\mathrm{CM}$. Among 
patients affected by $\mathrm{CM}$, acute medication overuse is about $30-50 \%$ and it is estimated that the medication overuse increases the risk of migraine progression sevenfold.

\section{Mechanisms of action}

Topiramate [2,3:4,5-Bis- $O$-(1-methylethylidene)- $\beta$-D-fructopyranose sulfamate] is a sulfamate-substituted monosaccaride, derived from D-fructose. Several molecular activities have been identified for topiramate: inhibitory effects on voltage-gated $\mathrm{Na}^{+}$and $\mathrm{Ca}^{2+}$ channels, modulation of glutamate-mediated neurotransmission (reducing depolarization induced by AMPA and kainate mediated currents), enhancement of some types of $\mathrm{GABA}_{\mathrm{A}}$ receptors, inhibition of carbonic anhydrase (CA); other less established mechanisms of action have been proposed, such as modulation of $\mathrm{K}^{+}$conductance and proteins regulating neurotransmitter release from synaptic terminals [19]. In brief, the drug reduces excitatory neurotransmission and enhances inhibitory neurotransmission, both probably involved in the pathophysiology of migraine. Topiramate, through a broad profile of action, might act at different levels: reducing nociceptive transmission through trigeminovascular modulation, and inhibiting cortical spreading depression [20, 21].

\section{The role of topiramate in migraine prevention}

Several large, randomized, placebo-controlled trials have proven topiramate to be effective for migraine prevention in adults [22-24], with $100 \mathrm{mg} /$ day being the target dose. Demonstration of efficacy was based on reduction in migraine frequency and use of acute medication, improvement of quality of life evaluated by the Migraine Specific Questionnaire (MSQ) and the Short-Form36 (SF36). The benefits appear after the first month of treatment and persist throughout the subsequent 6-month treatment period. Data from the open-label extension of pivotal trials and a successive randomized, placebo-controlled long-term study showed that benefits were sustained with prolonged treatment up to 12-14 months [25, 26].

Based on the analysis of a large database of $>1,500$ patients included in four randomised, double-blind, placebo-controlled trials, topiramate was generally safe and reasonably well tolerated in migraine prevention [27]. Most common adverse events (AE) associated with topiramate were mild or moderate in severity and occurred more frequently during titration. Paresthesia was the most common $\mathrm{AE}(35,51$, and $49 \%$ at 50, 100, and $200 \mathrm{mg} / \mathrm{die}$, respectively) which appeared to be self-limiting and not typically leading to drug discontinuation. Unlike other preventive treatments, topiramate was not associated with weight gain but rather with weight loss $(67 \%$ of patients with topiramate vs. $19 \%$ with placebo).

\section{Prevention of migraine progression}

A pooled analysis of data from the three pivotal trials suggested that preventive therapy with topiramate $100 \mathrm{mg} /$ day may reduce the risk of progression to chronic forms of headache [18]. This analysis shows that topiramate reduces the risk of an increase in headache rate; specifically, patients at higher risk of developing CM (those with $\geq 12$ headache days at baseline) appeared to benefit most from topiramate therapy. Furthermore, topiramate reduced the use of acute medication, particularly in patients with high frequency of migraine at baseline.

Data from open-label studies [28, 29] and one small placebo-controlled trial [30] suggested topiramate to be useful also in CM.

More recently, two multicenter, randomized, doubleblind, placebo controlled trials, one performed in the USA and the other one in Europe, both published in 2007, demonstrated that topiramate is effective and safe also in patients affected by $\mathrm{CM}$, in the presence or absence of medication overuse, and/or of other migraine prophylaxis [31, 32]. An integrated review of these studies was recently published by Silberstein and colleagues [33] describing differences in patient populations and in the classification of CM between the US and European trials. However, the key difference was the absence of limitation in acute medication intake, which allowed the European trial to demonstrate extended benefit of topiramate also to the subgroup of patients complicated by medication overuse.

\section{Conclusion}

Based on American and European guidelines [34, 35], the goals of a preventive treatment are to reduce frequency and severity of attacks, to improve response to acute therapy, to reduce use of acute medications, to limit disability, and to improve quality of life. When clinicians choose the best treatment for a patient they have to consider different aspects such as frequency of attacks, contraindications to therapies, adverse effects, and comorbidities.

Recent evidence suggests that migraine preventive treatment may also reduce the risk of transformation of episodic migraine to chronic headache. Therefore, a prophylaxis treatment should be initiated early once patients with risks of progression, i.e., elevated migraine frequency and/or frequent acute medication intake, have been identified. 
Topiramate, by acting positively on both these risk factors may prevent the development of CM. Furthermore, topiramate is effective in the treatment of patients already progressed to CM and difficult-to-treat conditions associated with medication overuse.

Based on its wide spectrum of mechanisms of action, topiramate may exert its effect in migraine prevention by reducing nociceptive transmission through trigeminovascular modulation, and inhibiting neuronal hyperexcitability, which affects initiation and development of CSD.

Topiramate represents an additional therapeutic option that is able to act at different levels of the migraine cycle: reduction of frequency in episodic migraine, prevention, and treatment of CM.

\section{Conflict of interest None.}

\section{References}

1. Welch KMA, Nagesh V, Aurora SK et al (2001) Periaqueductal gray matter dysfunction in migraine: cause or the burden of illness? Headache 41:629-637

2. Bigal ME, Lipton RB (2008) The chronification of headache. Concepts and mechanisms of migraine chronification. Headache 48:7-15

3. Leão AAP (1944) Spreading depression of activity in cerebral cortex. J Neurophysiol 7:359-390

4. Kunkler PE, Kraig RP (2003) Hippocampal spreading depression bilaterally activates the caudal trigeminal nucleus in rodents. Hippocampus 13:835-844

5. Lauritzen M, Jorgensen MB, Diemer NH et al (1982) Persistent oligoemia of rat cerebral cortex in the wake of spreading depression. Ann Neurol 12:469-474

6. Leao A, Morison R (1945) Propagation of spreading cortical depression. J Neurophysiol 8:33-45

7. Milner PM (1958) Note on a possible correspondence between the scotomas of migraine and spreading depression of Leao. Electroencephalogr Clin Neurophysiol Supp 110:705

8. Cao Y, Welch KM, Aurora S et al (1999) Functional MRI-BOLD of visually triggered headache in patients with migraine. Arch Neurol 56:548-554

9. Hadjikhani N, Sanchez Del Rio M, Wu O et al (2001) Mechanisms of migraine aura revealed by functional MRI in human visual cortex. Proc Natl Acad Sci USA 98:4687-4692

10. Ayata C, Jin H, Kudo C et al (2006) Suppression of cortical spreading depression in migraine prophylaxis. Ann Neurol 59:652-661

11. Kruit MC, van Buchem MA, Hofman PA et al (2004) Migraine as a risk factor for subclinical brain lesions. JAMA 291:427-434

12. Lipton RB, Pan J (2004) Is migraine a progressive brain disease? JAMA 291:493-494

13. Granziera C, DaSilva AFM, Snyder J et al (2006) Anatomical alterations of the visual motion processing network in migraine with and without aura. PLoS Med 3(10):e402. doi:10.1371/ journal.pmed.0030402

14. Montagna P, Cevoli S, Marzocchi N et al (2003) The genetics of chronic headaches. Neurol Sci 24(suppl 2):S51-S56

15. Welsh KMA, Goadsby PJ (2002) Chronic daily headache. Curr Opin Neurol 115:287-295
16. Kitaj MB, Klink M (2005) Pain thresholds in daily transformed migraine versus episodic migraine headache patients. Headache 45:992-998

17. Scher AI, Stewart WF, Ricci JA, Lipton RB (2003) Factors associated with the onset and remission of chronic daily headache in a population-based study. Pain 106:81-89

18. Limmroth V, Biondi D, Pfeil J et al (2007) Topiramate in patients with episodic migraine: reducing the risk for chronic forms of headache. Headache 47:13-21

19. Shank RP, Maryanoff BE (2008) Molecular pharmacodyynamics, clinical therapeutics, and pharmacokinetics of topiramate. CNS Neurosci Ther 14:120-142

20. Akerman S, Goadsby PJ (2005) Topiramate inhibits cortical spreading depression in rat and cat: impact in migraine aura. Neuroreport 16:1383-1387

21. Storer RJ, Goadsby PJ (2004) Topiramate inhibits trigeminovascular neurons in the cat. Cephalalgia 24:1049-1056

22. Brandes JL, Saper JR, Diamond M et al (2004) MIGR-002 Study Group. Topiramate for migraine prevention: a randomized controlled trial. JAMA 291:965-973

23. Diener HC, Tfelt-Hansen P, Dahlof C et al (2004) Topiramate in migraine prophylaxis-results from a placebo-controlled trial with propranolol as an active control. J Neurol 251:943-950

24. Silberstein SD, Neto W, Schmitt J et al (2004) Topiramate in migraine prevention: results from a large controlled trial. Arch Neurol 61:490-495

25. Rapoport A, Mauskop A, Diener HC et al (2006) Long-term migraine prevention with topiramate: open-label extension of pivotal trials. Headache 46:1151-1160

26. Diener HC, Agosti R, Allais G et al (2007) Cessation versus continuation of 6-month migraine preventive therapy with topiramate (PROMPT): a randomised, double-blind, placebocontrolled trial. Lancet Neurol 6:1054-1062

27. Adelman J, Freitag FG, Lainez M et al (2008) Analysis of safety and tolerability data obtained from over 1,500 patients receiving topiramate for migraine prevention in controlled trials. Pain Med 9:175-185

28. Mosek A, Dano M (2005) Topiramate in the treatment of refractory chronic daily headache: an open trial. J Headache Pain 6:77-80

29. Bartolini M, Silvestrini M, Taffi R et al (2005) Efficacy of topiramate and valproate in chronic migraine. Clin Neuropharmacol 28:277-279

30. Silvestrini M, Bartolini M, Coccia M et al (2003) Topiramate in the treatment of chronic migraine. Cephalalgia 23:820-824

31. Silberstein SD, Lipton RB, Dodick DW et al (2007) Efficacy and safety of topiramate for the treatment of chronic migraine: a randomized, double-blind, placebo-controlled trial. Headache $47: 170-180$

32. Diener H-C, Bussone G, Van Oene JC et al (2007) Topiramate reduces headache days in chronic migraine: a randomized, double-blind, placebo-controlled study. Cephalalgia 27:814-823

33. Silberstein S, Diener HC, Lipton R et al (2008) Epidemiology, risk factors, and treatment of chronic migraine: a focus on topiramate. Headache 48:1087-1095

34. Ramadan NM, Silberstein SD, Freitag FG et al (2000) Evidencebased guidelines for migraine headache in the primary care setting: pharmacological management for prevention of Headache 597 migraine. Am Acad Neurol, pp 1-55. Available at http:// www.aan.com/professionals/practice/pdfs/g10090.pdf. Accessed 4 June 2006

35. Evers S, Afra J, Frese A et al (2006) EFNS guideline on the drug treatment of migraine-report of an EFNS task force. Eur J Neurol 13:560-572 